\title{
OCRM: Optimal Cost Region Matching Similarity Measure for Region Based Image Retrieval
}

\author{
N. Gnaneswara Rao ${ }^{1}$ T. Sravani ${ }^{2}$ and V. Vijaya Kumar $^{3}$ \\ ${ }^{1}$ Department of CSE, Vignan University, Vadlamudi, A.P., India \\ ${ }^{2}$ Software Engineer, Visakhapatnam \\ ${ }^{3}$ Dept. of CSE/IT, Anurag Group of Institutions, Ghatkesar, JNT University • \\ Hyderabad, A.P., India \\ gnani.nitta@gmail.com,26sravani@gmail.com,vijayvakula@gmail.com.
}

\begin{abstract}
Content Based Image Retrieval (CBIR) has been the most significant area in the applications of Pattern recognition and Computer Vision for the tast three decades. However, there are many open problems left unresolved. Among these, one of the current problems of CBIR is to obtain an effective Similarity Measure The CBIR systems make use of Integrated Region Matching (IRM) to match segmented inages which is computationally economic, but it is not a metric distance whereas systems that (1se) Minimum Cost Region Matching (MiCRoM) as a similarity measure is a metric distance, but computationally expensive. In order to address the above problem, this paper has developed the Optimal Cost Region Matching (OCRM) similarity measure for region based image retrieval. The proposed OCRM uses the north-west Gorner rule of the Transportation problem that fulfills the monge property. The experiment carred out bn 1000 color images taken from the Corel database that are compared with TRM, and MiCRoM similarity measures.
\end{abstract}

Keywords: IRM, MiCRoM, CBR, OCRM, Similarity Measure, Transportation problem, Monge condition

\section{Introduction}

Since 1970`s, the Content Based Image Retrieval (CBIR) has been playing a vital role in the image database management and image retrieval. There is a fast progress in the computer speed and space with the decline in the memory cost, due to which large number of image database are used in various applications like medicine, entertainment, satellite, biometric etc. This in turn made the necessity to search for the image in the image database. The CBIR system is playing an important role in the retrieval of images from large databases $[1,2,3$, 26]. This system [4] examines the images from the database by utilizing pictorial indications. It is well capable and efficiently developed for retrieving images pertaining to the speed and precision. Thus, the system extracts the features from the Query and Target images and measure the similarity among these images based on given features. In Order to represent an image, there are many recent approaches $[4,12,20,21,22]$ like segmenting the image into a number of regions with the goal to extract the objects in the image. Until now, no supervised segmentation algorithm introduced, to obtain an accurate segmentation and good reasonable results.

The motivation for this paper is to obtain similarity measures [5, 6, 24, 25] for the images that is both optimal and computationally economic. The Integrated Region Matching (IRM) 
[5] is a Similarity Measure for region based image comparison. To utilize the maximum information about an image, IRM integrates the properties of all segmented regions. With each image segmentation, the features like color, shape, size, and the spatial position of the acquired regions extracted. Then the regions matched using the Integrated Region Matching (IRM) similarity measure. This distance measure is not a metric so it cannot use the metric access structures or filtering techniques.

Since the IRM matches the individual regions. The main objective of the similarity distance is to match segmented images. Even though the solutions obtained with every specific regions of image combined, the complete content of the image cannot be compared. To overcome this, the Minimum Cost Region Matching (MiCRoM) metric distance method [6] is used. MiCRoM is a region based Image Retrieval (IR) approach, to match the contents of segmented images. The main objective of MiCRoM is to measure the similarity using the minimum-cost network flow problem for the target and query image. [7, 8, 23]. The use of metric measures is to decrease the search space and number of cômparions and make use of Metric Access methods.

\section{Proposed Methodology}

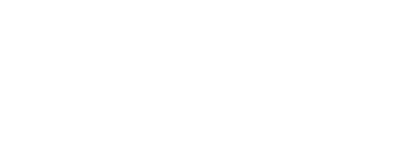

In the proposed Content Based Image Retrieval CBIR System, feature extraction and representation is the first and foremost step. Then the image partitioned in terms of the fixed number of blocks. Based on the fixed number of blocks, this paper proposed a novel scheme called Optimal Cost Region Matching (OGRM) for the elfective and efficient image retrieval that satisfies the human perception (in) an accurate way. The entire process represented in Figure 1 and explained in detail.

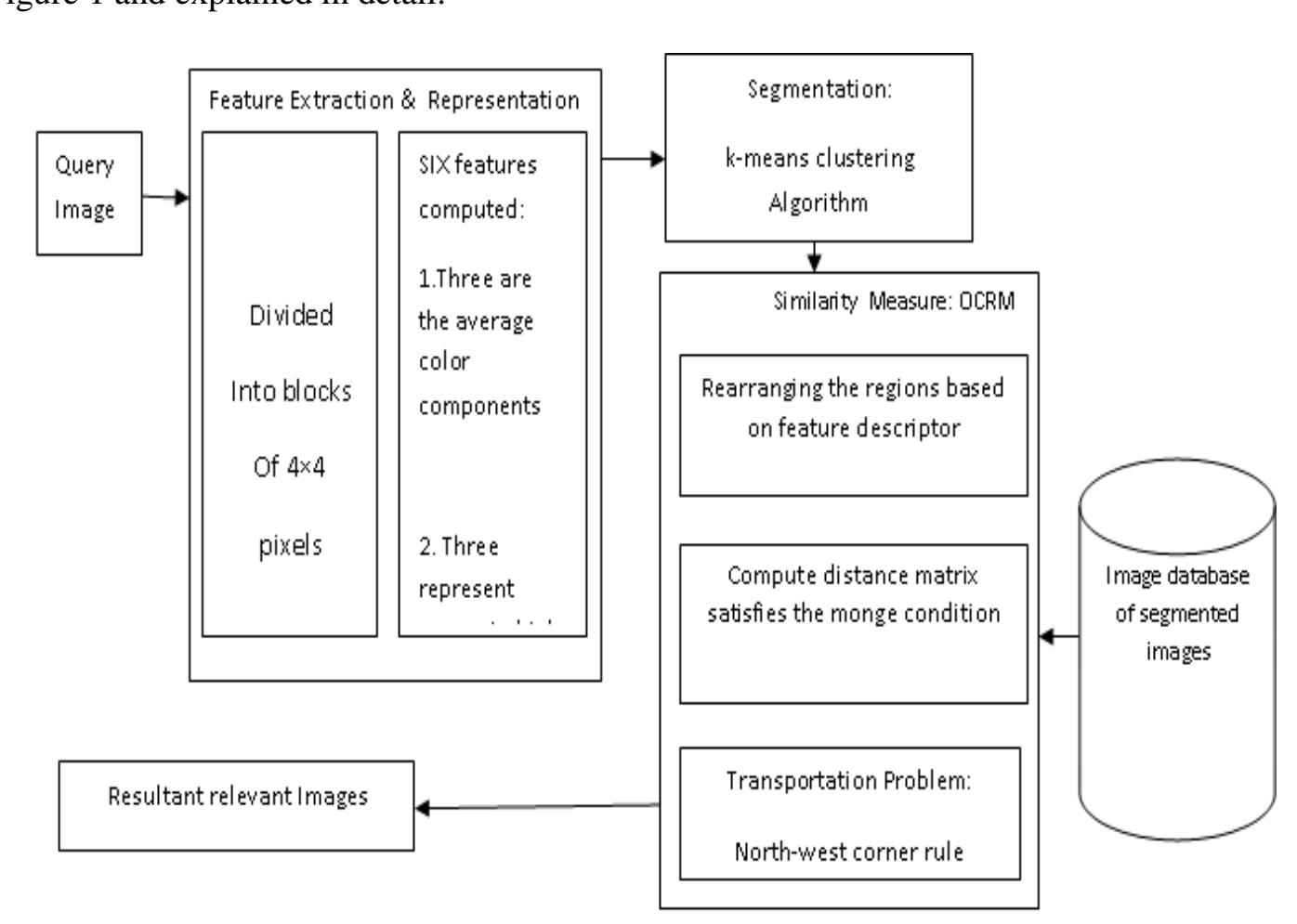

Figure 1. The OCRM similarity measure 


\subsection{Feature Extraction and Representation}

In the first step, the image divided into blocks of $4 \times 4$ pixels and obtained the feature vector for every block. This block size considered to enhance the effectiveness of color and texture features. The proposed methodology computes six features for each and every block in which three of them are the usual color components in a $4 \times 4$ block and the other three signify dynamism in high occurrence groups of the wavelet transforms[10,16],i.e. the square root of the second order instant of wavelet coefficients. The LUV color space [14, 15] mentioned in this paper, to extract the color components where L encrypts luminance; $U$ and $\mathrm{V}$ encrypt color information (chrominance). The other three features acquired by applying Daubechies-4 wavelet alter to the L component of the image. After one-level wavelet transformation, the $4 \times 4$ block disintegrated into four frequency bands: LL, LH, $\mathrm{HL}$ and $\mathrm{HH}$ bands. Each band contains $2 \times 2$ coefficients such as $C_{k=1} C_{k n+1} C_{k+1]}$ and $C_{k+1}$ are the coefficients of wavelet bands $\mathrm{LH}, \mathrm{HL}$ and $\mathrm{HH}$ ). Equation (1) computes the texture feature fo as follows:

$$
f_{b}=\sqrt{\frac{C_{k, l}^{2}+C_{k, l+1}^{2}+C_{k+1, l}^{2}+C_{k+1, l+1}^{2}}{4}}
$$

Where $b$ is one of the LH, HL, and HH bands (The inspiration for using the features, mined from high frequency bands is that they reproduce texture propeyties in a proficient way.

\subsection{Segmentation}

The next step in the proposed method is to segment the image in terms of blocks having 64 regions depending on extracted colbr and texture features, which resemble to the substances present in the image. Here the number of regions fixed to satisfy the properties of monge matrix. The segmentation of mages based on color and frequency features using the k-mean's algorithm [13, 17, 18, 19]. The k-means algorithm segment, the feature vectors into numerous groups where every group corresponds to one region of the segmented image. One of the foremost advaptages for using the kemeans clustering algorithm for dissection is that the blocks in each region need no be neighborhood blocks.

\subsection{OCRM: Optimal Cost Region Matching Similarity Measure}

The proposed OCRM derives a distance matrix that satisfies the monge condition between Region descriptors. For the Distance Matrix, a novel similarity measure derived based on the transportation problem.

2.3. Computation of Monge Distance Matrix: The Region Descriptors computed, after segmentation from features of the respected regions. Then the regions sorted in an ascending order based on the region descriptor. There will be a rearrangement in the regions and then the distance calculated between the query image and the target image depending on the equation (2).

$$
d_{i j j}=\left\|q_{i}-t_{i}\right\|
$$

Where $d_{\mathrm{h} j \mathrm{j}}$ is the distance between the region descriptor of the query image and target image $q_{i j}$ is the region descriptor of the $i^{\text {th }}$ region of the query image and $t_{j}$ is the region descriptor of the $i^{\text {th }}$ region of the target image 


$$
d_{i, j}+d_{i+1, j+1} \leq d_{i, j+1}+d_{i+1, j}
$$

According to monge property, Equation 3 is always true. Therefore, the $d_{i, j}$ obtained after the rearrangement in the regions in the sorted order always satisfies the monge property.

Proof:

Assume, $\quad d_{i j}=\left|q_{i j}-t_{j}\right|$

We have to show that,

$$
d_{i j}+d_{i+1, j+1} \leq d_{i, j+1}+d_{i+1} f^{2}
$$

Note that, $q_{i}<q_{i+1} \quad$ and $t_{j}<t_{j+1}$

Since we have sorted the array

Case 1: $q_{i} \leq t_{j} \leq q_{i+1} \leq t_{j+1}$

Following defined as: $d_{i j}=t_{j}-q_{i}$

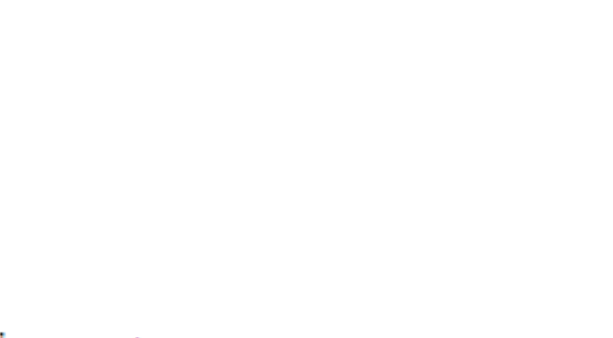

Therefore,

$d_{i j}+d_{i+1, j+1}=t_{j}-\left(q_{i}\left(-t_{i j+1}-q_{i+1}\right)\left(t-t_{i+1}-q_{i j}+t_{j+1}\right.\right.$

$d_{i j+1}+d_{i+1 j i}=t_{j+1}-q_{i j}+q_{i+1}^{0} S_{i j}=q_{i+1}-t_{j}-q_{i j}+t_{j+1}$

Since in this case $q_{i+1}$ (t)

We have $d d_{i f}+d_{i+1, j+1} \leq d_{i, j+1}+d_{i+1}$ as required

Similarly, there arefive more cases

Case 2: $4-q_{i+1} \leq t_{j} \leq t_{j+1}$

Case $3 \bigcirc-q_{i} \leq t_{j+1} \leq q_{i+1}$

Case 4. $\quad t_{j} \leq t_{j+1} \leq q_{i} \leq q_{i+1}$

Case 5: $\quad \mathrm{t}_{j} \leq q_{\mathrm{i}} \leq q_{\mathrm{i}+1} \leq \mathrm{t}_{j+1}$

Case 6: $\quad q_{i} \leq t_{j} \leq t_{j+1} \leq q_{i+1}$

In all these cases, easily shown that

$d_{i, j}+d_{i+1, j+1} \leq d_{i, j+1}+d_{i+1} j$

Therefore, the matrix thus generated is a Monge Matrix.

Finally, similarity measure is the essential metric in the course of image retrieval, to choose the effectiveness and efficiency of retrieval techniques. A new similarity measure based on the transportation problem called North-West Corner rule, proposed for the distance matrix. The North-West Corner rule, the Lowest Cost Entry rule, and Vogel's method are used for finding the initial feasible solution of the transportation problem. When the distance matrix satisfies the monge property, the North-West Corner rule is the method that gives the optimal solution with less computational time. This rule provides an optimum solution of the 
transportation problem for all source and demand vectors ' $\mathrm{a}$ ' and ' $\mathrm{b}$ ' with $\sum_{i=1}^{m} a_{i}=\sum_{j=1}^{n} b_{j}$ only if the cost matrix, $\mathrm{C}$ is a Monge Matrix [8,9,11]. Given $m \times n$ cost matrix $\mathrm{C}$ with items from $\mathrm{R}$, a nonnegative source vector $a=\left(a_{10 \ldots} \ldots a_{\mathrm{m}}\right)$ and a nonnegative demand vector $b=\left(b_{10 \times \cdots}=b_{\mathrm{m}}\right)$ such that $\sum_{i=1}^{m} a_{i}=\sum_{j=1}^{n} b_{j}$, the Classical Hitchcock Transportation Problem (TP) can formulated as follows:

$$
\begin{gathered}
\min \sum_{i=1}^{m} \sum_{j=1}^{n} c_{i j} x_{i j} \\
\text { s.t. } \sum_{j=1}^{n} x_{i j}=a_{i} \quad \text { for all } i=1, \ldots ., m, \\
\sum_{i=1}^{m} x_{i j}=b_{j} \quad \text { for all } \quad j=1, \ldots . ., n, \\
x_{i j \geq 0} \quad \text { for all } i, j .
\end{gathered}
$$

An example of a test case images having five regions shown. The Table 1 and 2 shows the region descriptor values and area size of the query and target images. The distance matrix obtained from the region descriptor of the query mage and target images shown in the Table 3. The distances computed for the Table 8 using IRM.MiCRoM, and the North-west corner method listed in Table 4. From the Table 4, it is evident that the MiCRoM similarity measure using Lowest Cost entry rule gives the optimal distance.

The Table 5 and 6 shows the region descriptor values after sorting by the proposed method of query and target images respectively. The Table 7 shows the distance matrix formed by the proposed method, which satusfies the monge property. The distances computed from the Table 7 using IRM, MiCRoM, and Optimal Cost Region Matching (OCRM) similarity measure using the North-west Corner method listed in Table 8. From the Table 8, it is evident that the optimal solution obtained from MiCRoM and OCRM similarity measures. The MiCRoM yielded the optimal solution after three iterations whereas the proposed OCRM yield the same optimal solution with the single iteration. Therefore, the proposed method is better than the other methods.

Table 1. The query image: regions, Region descriptors, and areas

\begin{tabular}{|l|l|l|l|l|l|l|}
\hline Regions & $\mathbf{1}$ & $\mathbf{2}$ & $\mathbf{3}$ & $\mathbf{4}$ & $\mathbf{5}$ \\
\hline Descriptors & 8 & 6 & 7 & 2 & 8 \\
\hline Areas & 0.15 & 0.1 & 0.1 & 0.3 & 0.35 \\
\hline
\end{tabular}

Table 2. Target image: regions, region descriptors and areas

\begin{tabular}{|c|c|c|c|c|c|}
\hline Regions & $\mathbf{1}$ & $\mathbf{2}$ & $\mathbf{3}$ & $\mathbf{4}$ & $\mathbf{5}$ \\
\hline Descriptors & 9 & 2 & 7 & 3 & 1 \\
\hline Areas & 0.25 & 0.2 & 0.15 & 0.2 & 0.2 \\
\hline
\end{tabular}


Table 3. The Distance matrix of the query image and target image

\begin{tabular}{|c|c|c|c|c|c|}
\hline regions & $\mathbf{1}$ & $\mathbf{2}$ & $\mathbf{3}$ & $\mathbf{4}$ & $\mathbf{5}$ \\
\hline $\mathbf{1}$ & 1 & 6 & 1 & 5 & 7 \\
\hline $\mathbf{2}$ & 3 & 4 & 1 & 3 & 5 \\
\hline $\mathbf{3}$ & 2 & 5 & 0 & 4 & 6 \\
\hline $\mathbf{4}$ & 7 & 0 & 5 & 1 & 1 \\
\hline $\mathbf{5}$ & 1 & 6 & 1 & 5 & 7 \\
\hline
\end{tabular}

Table 4. Distances between query and target images

\begin{tabular}{|c|c|c|}
\hline $\begin{array}{c}\text { Similarity } \\
\text { measure }\end{array}$ & Method & Distance $(\mathrm{Q}, \mathrm{T})$ \\
\hline IRM & $\begin{array}{c}\text { Lowest cost } \\
\text { entry rule }\end{array}$ & \\
\hline MiCRoM & $\begin{array}{l}\text { Optimal solut } \\
\text { of Lowest co } \\
\text { entry rule }\end{array}$ & \\
\hline - & $\begin{array}{l}\text { North-west } \\
\text { Corner Meth }\end{array}$ & \\
\hline
\end{tabular}

Table 5. Query image: regions, region descriptors and areas

\begin{tabular}{|c|c|c|c|c|c|}
\hline Regions & 1 & 2 & 3 & 4 & 5 \\
\hline Descriptors & 2 & 6 & 7 & 8 & 8 \\
\hline Areas & 0.3 & 0.1 & 0.1 & 0.15 & 0.35 \\
\hline
\end{tabular}

Table 6. Target iniage: regions, region descriptors and areas

\begin{tabular}{|c|c|c|c|c|c|}
\hline Regions & 1 & 2 & 3 & 4 & 5 \\
\hline Descriptors & 1 & 2 & 3 & 7 & 9 \\
\hline Areas & 0.2 & 0.2 & 0.2 & 0.15 & 0.25 \\
\hline
\end{tabular}

able 7. The Distance matrix of the query image and target image

\begin{tabular}{|c|c|c|c|c|c|}
\hline regions & $\mathbf{1}$ & $\mathbf{2}$ & $\mathbf{3}$ & $\mathbf{4}$ & $\mathbf{5}$ \\
\hline $\mathbf{1}$ & 1 & 0 & 1 & 5 & 7 \\
\hline $\mathbf{2}$ & 5 & 4 & 3 & 1 & 3 \\
\hline $\mathbf{3}$ & 6 & 5 & 4 & 0 & 2 \\
\hline $\mathbf{4}$ & 7 & 6 & 5 & 1 & 1 \\
\hline $\mathbf{5}$ & 7 & 6 & 5 & 1 & 1 \\
\hline
\end{tabular}


Table 8. Distances between query and target images

\begin{tabular}{|c|c|c|}
\hline $\begin{array}{c}\text { Similarity } \\
\text { measure }\end{array}$ & Method & Distance(Q,T) \\
\hline IRM & $\begin{array}{c}\text { Lowest cost } \\
\text { entry rule }\end{array}$ & 2.0 \\
\hline MiCRoM & $\begin{array}{c}\text { Optimal } \\
\text { solution of } \\
\text { Lowest cost } \\
\text { entry rule }\end{array}$ & $\mathbf{1 . 9}$ \\
\hline OCRM & $\begin{array}{c}\text { North-west } \\
\text { Corner Method }\end{array}$ & $\mathbf{1 . 9}$ \\
\hline
\end{tabular}

\section{Experimental Results}

The proposed OCRM tested with the general-purpose inage database from COREL consisting of nearly 1000 images. These images are stored in JPEG format with size $384 \mathrm{x}$ 256 or $256 \times 384$. According to the k means clustering algorithm/each image obtains 64 Regions with 6 features for each cluster. When aquery image is given, its features computed and the image segmented into 64 Regions. The segmented query image then compared against each of the segmented images stored in the database using IRM, MiCRoM and the proposed OCRM methods. The ten closest images from the database retrieved as similar images to the query image.

The Figures 2, 3 and 4 show the results of IRM, MiCRoM and OCRM methods respectively for top ten retrieved images for the query image \#200 (Building image). The Table 9 shows the results of the query image $\$ 200$ in the recovery process. The Table 10 and Figures 5, 6 shows Precision $v_{s}$ a number of images returned and Precision $v_{s}$ recall plots for the query image \#200. The retrieved results in the Table 9, 10 and Figures 5, 6 show that the OCRM and MiCRoM gives the equal results whereas IRM gives less precision and recall value for top 5,10 and 20 images

The Figures 7,8 and 9 show the results of IRM, MiCRoM and OCRM similarity measures for 10 retrieved images respectively of the query image \#524(Elephant image). The Table 11 shows the results for the query image \#524 in the retrieval process. The Table 12 and Figures 10, 11 shows Precision $\mathrm{v}_{\mathrm{s}}$ a number of images returned and Precision $\mathrm{v}_{\mathrm{s}}$ recall plots for the query image \#524. The retrieved results in the Table 11, 12 and Figures 10, 11 show that the OCRM and MiCRoM gives the equal results whereas IRM gives less precision and recall value for top 5 and 10 images.

The Figures 12, 13 and 14 show the results of IRM, MiCRoM and OCRM methods for 10 retrieved images respectively of the query image \#618(Flower image). The Table 13 shows the results for the query image \#618 in the retrieval process. The Table 14 and Figures 15, 16 shows Precision $\mathrm{v}_{\mathrm{s} \text {. }}$ a number of images reverted and precision $\mathrm{v}_{\mathrm{s}}$ recall plots for the query image \#618.

Finally, the results retrieved in the Table 13, 14 and Figures 15, 16 represent that IRM, MiCRoM and OCRM retrieve same result for top 5 and 10 images whereas for top 20 images, IRM gives comparatively less precision and recall value compared to MiCRoM and OCRM. 


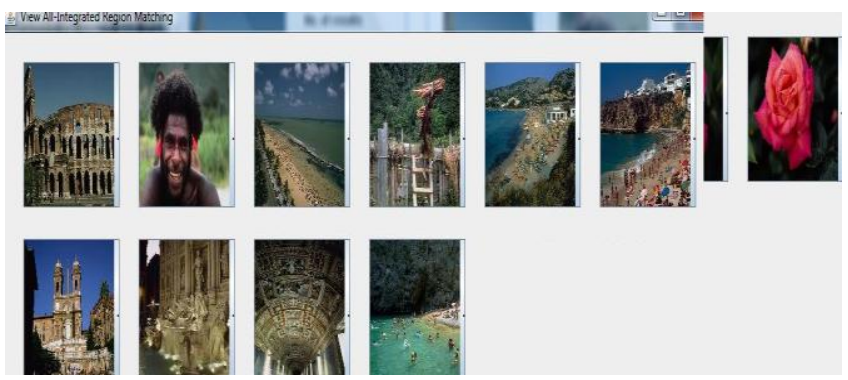

Figure 2. The ten relevant images of the query image \#200, using IRM

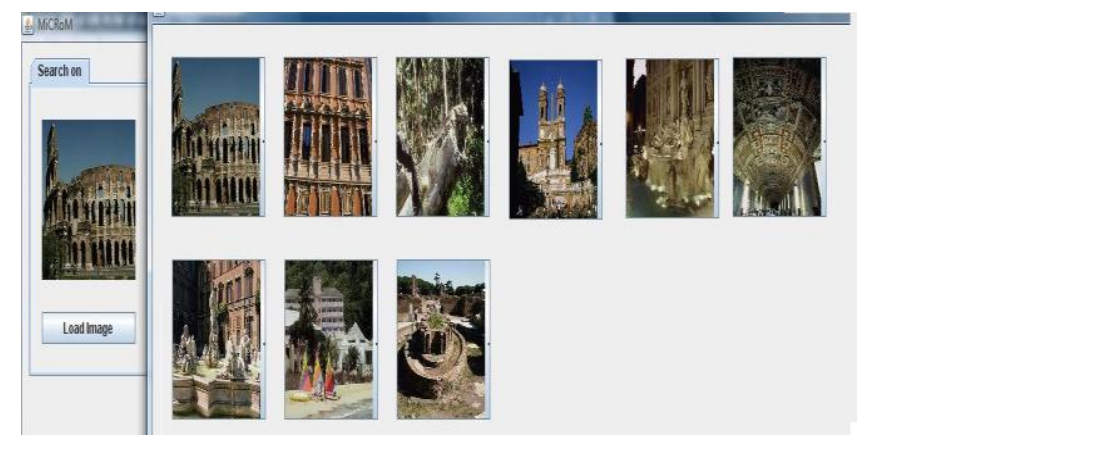

Figure 3. The ten relevant images of the query image \#200, using MiCRoM

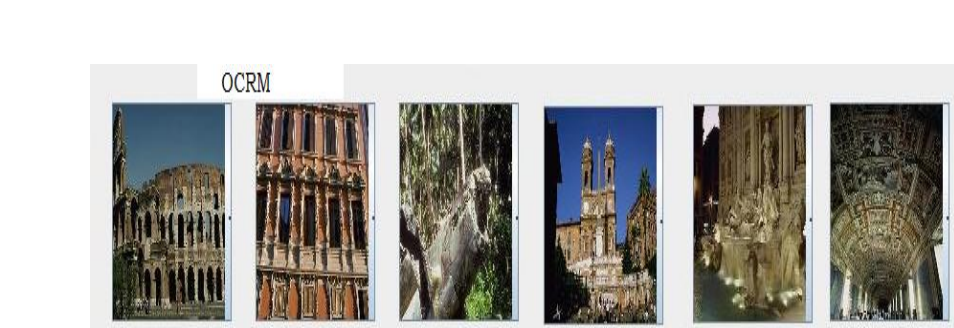

Figure 4. The ten relevant images of the query image \#200, using OCRM.

Table 9. The Retrieval results of the query image \#200

\begin{tabular}{|c|c|c|c|}
\hline $\begin{array}{c}\text { Query } \\
\text { \#200 }\end{array}$ & IRM & MiCRoM & OCRM \\
\hline 5 & 1 & 4 & 4 \\
\hline 10 & 4 & 8 & 8 \\
\hline 20 & 6 & 13 & 13 \\
\hline
\end{tabular}


Table 10. The precision of image \#200

\begin{tabular}{|c|c|c|c|}
\hline $\begin{array}{c}\text { Query } \\
\text { \#200 }\end{array}$ & IRM & MiCRoM & OCRM \\
\hline 5 & 0.2 & 0.8 & 0.8 \\
\hline 10 & 0.4 & 0.8 & 0.8 \\
\hline 20 & 0.3 & 0.65 & 0.65 \\
\hline
\end{tabular}

\section{Precision for Building Imáge}

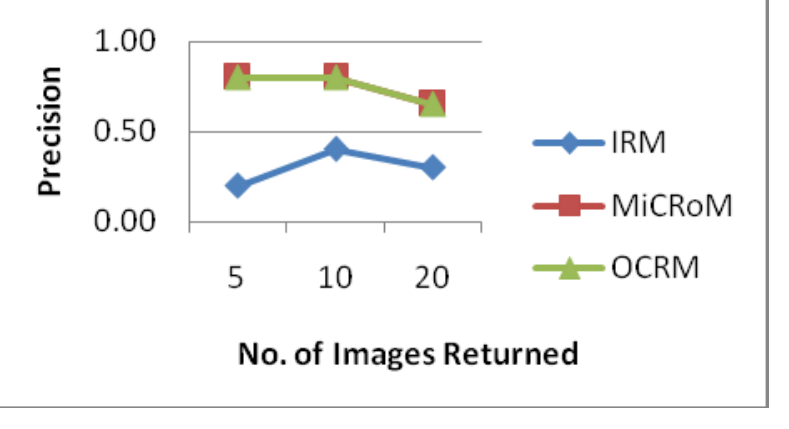

Figure 5. The Precision for the query image \#200

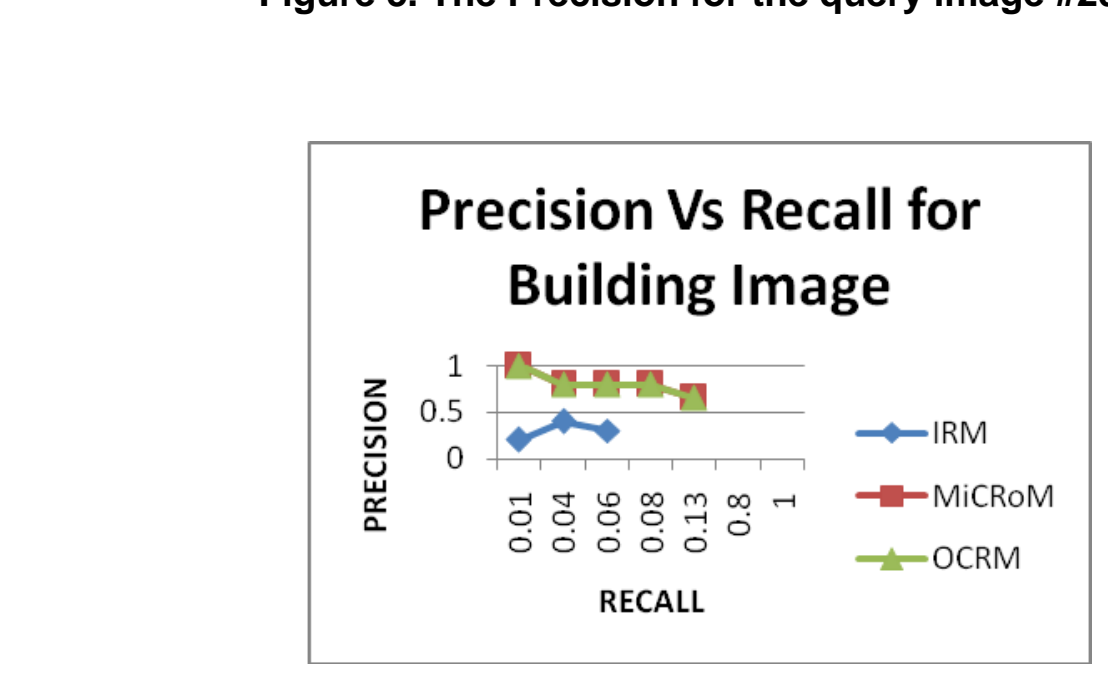

Figure 6. The Precision vs. Recall for the query image \#200(Building) 
International Journal of Multimedia and Ubiquitous Engineering

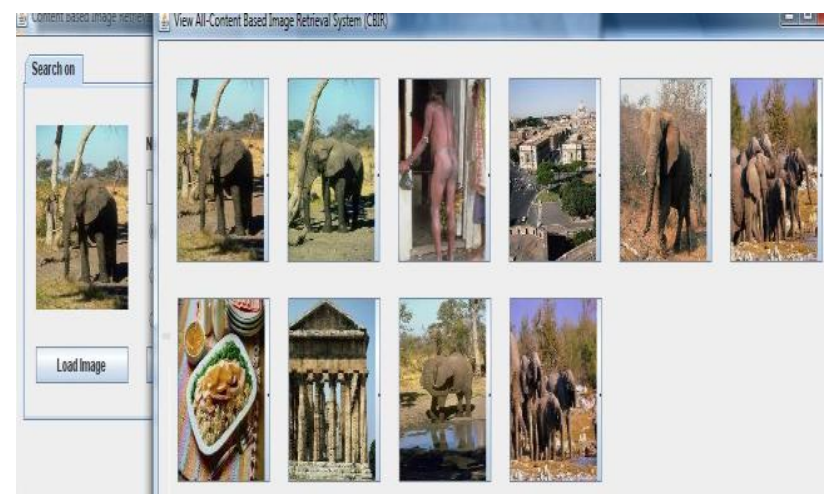

Figure 7. The ten relevant images of the query image \#524, using IRM

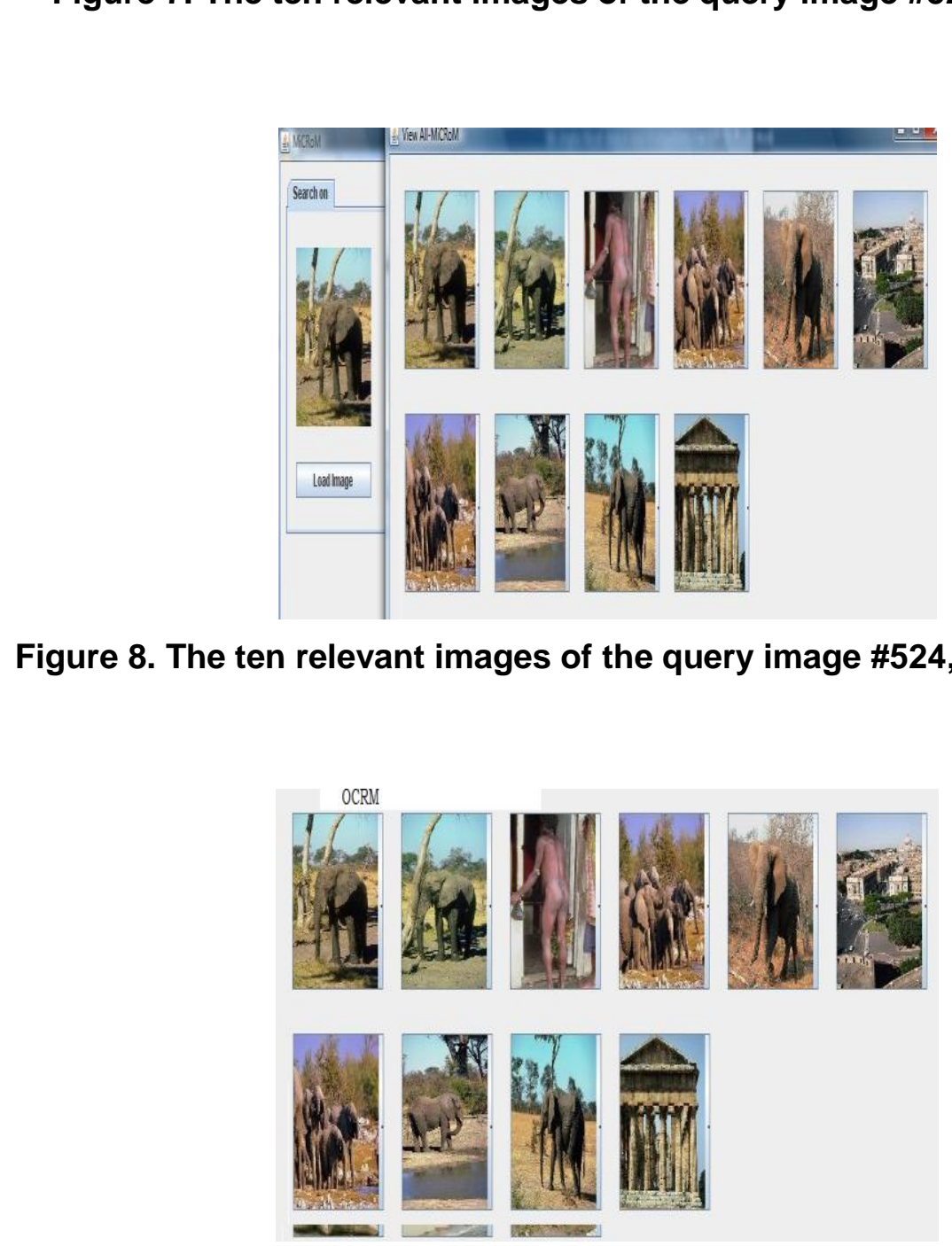

Figure 9. The ten relevant images of the query image \#524, using OCRM 
Table 11. The Retrieval results of the query image \#524

\begin{tabular}{|c|c|c|c|}
\hline $\begin{array}{c}\text { Query } \\
\text { \#524 }\end{array}$ & IRM & MiCRoM & OCRM \\
\hline 5 & 3 & 4 & 4 \\
\hline 10 & 6 & 7 & 7 \\
\hline 20 & 13 & 13 & 13 \\
\hline
\end{tabular}

Table 12. The Precision of image \#524

\begin{tabular}{|c|c|c|c|}
\hline $\begin{array}{c}\text { Query } \\
\text { \#524 }\end{array}$ & IRM & MiCRoM & OCRM \\
\hline 5 & 0.6 & 0.8 & 08 \\
\hline 10 & 0.6 & 07 & 0.7 \\
\hline 20 & 0.65 & 0.65 & 0.65 \\
\hline
\end{tabular}

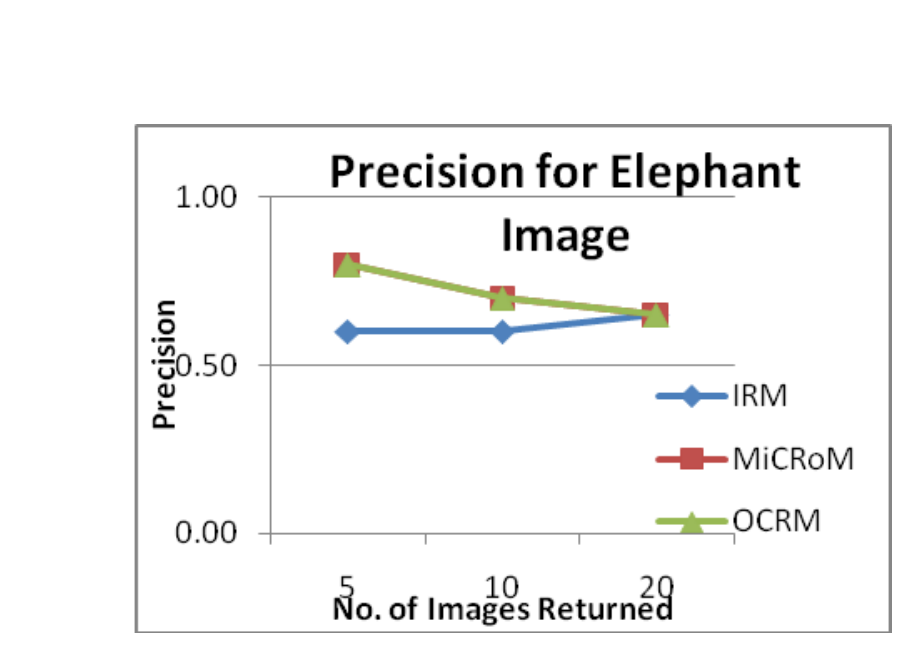

Figure 10. The Precision for query image \#524

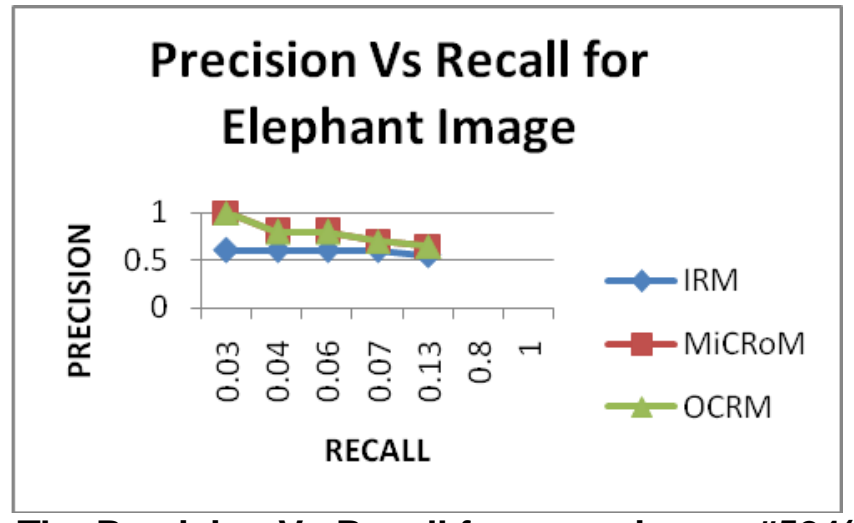

Figure 11. The Precision Vs Recall for query image \#524(Elephant) 


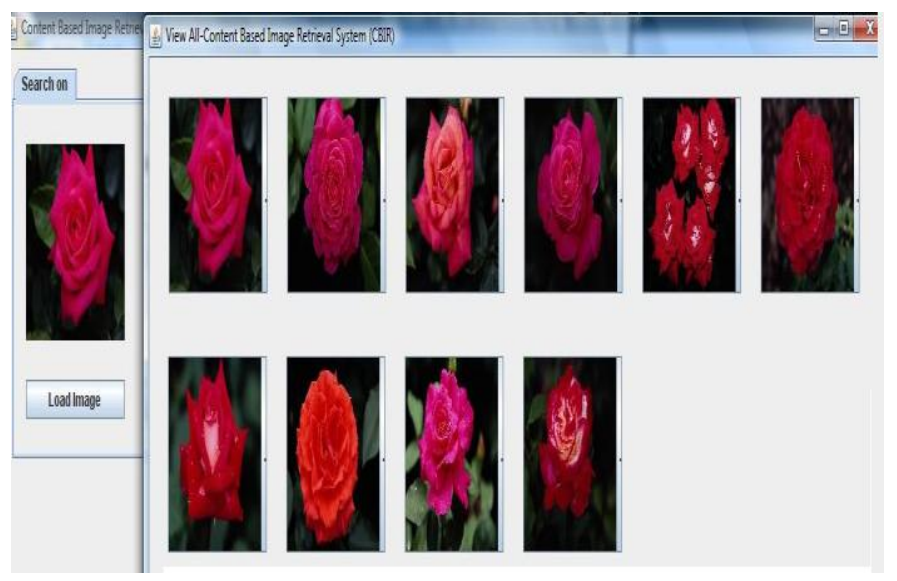

Figure 12. The ten relevant images of the query imaige \#618, USing IRM

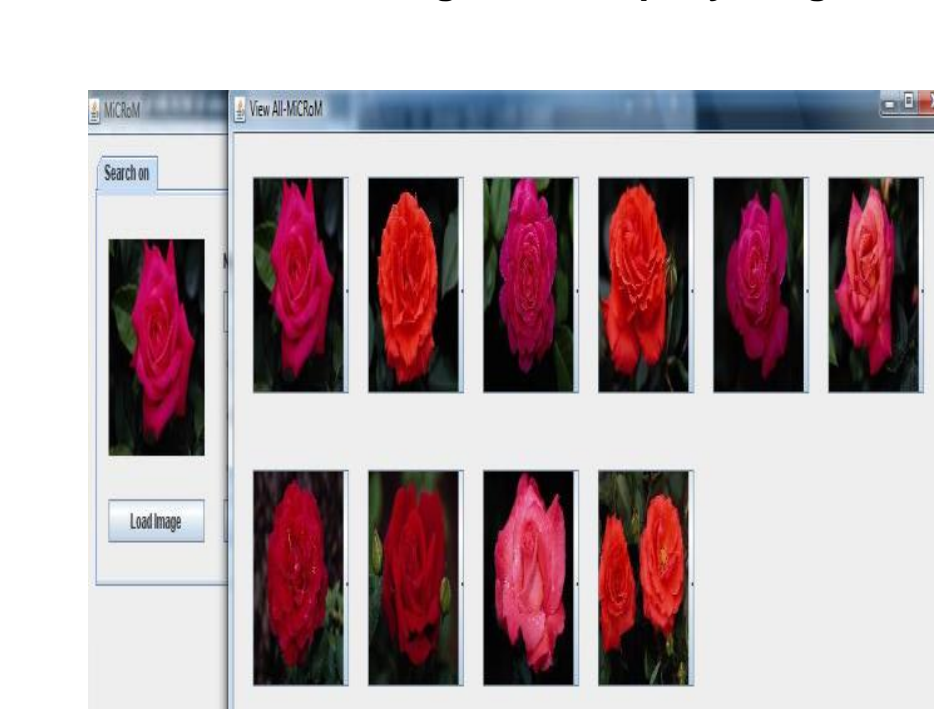

Figure 13. The ten relevant images of the query image\# 618, using MiCRoM

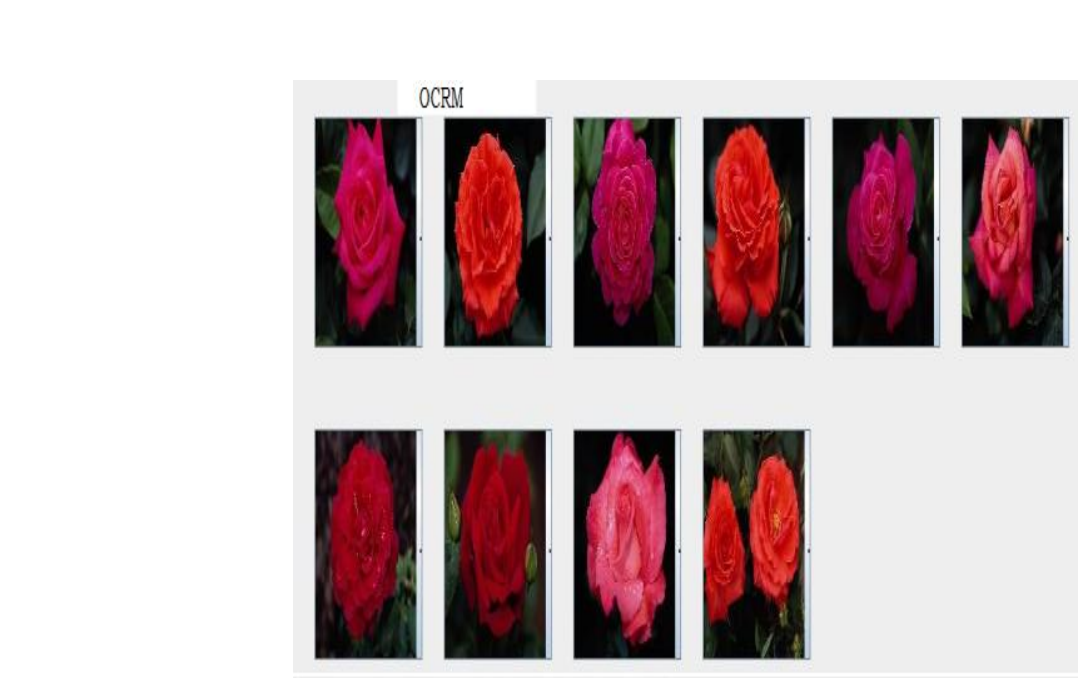

Figure 14. The ten relevant images of the query image \#618, using OCRM 
Table 13. The Retrieval results of the query image \#618

\begin{tabular}{|c|c|c|c|}
\hline $\begin{array}{c}\text { Query } \\
\text { \#618 }\end{array}$ & IRM & MiCRoM & OCRM \\
\hline 5 & 5 & 5 & 5 \\
\hline 10 & 10 & 10 & 10 \\
\hline 20 & 18 & 20 & 20 \\
\hline
\end{tabular}

Table 14. The Precision of image \#618

\begin{tabular}{|c|c|c|c|}
\hline $\begin{array}{c}\text { Query } \\
\text { \#618 }\end{array}$ & IRM & MiCRoM & OCRM \\
\hline 5 & 1 & 1 \\
\hline 10 & 1 & \\
\hline 20 & 0.9 & & 1 \\
\hline
\end{tabular}

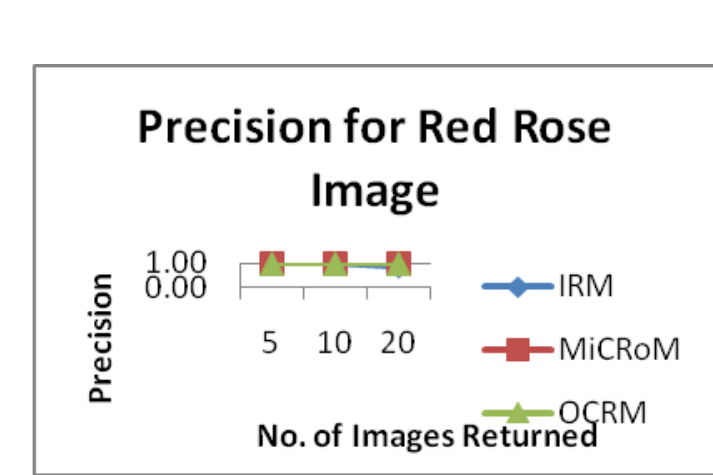

Figure 15. The Precision for the query image \#618

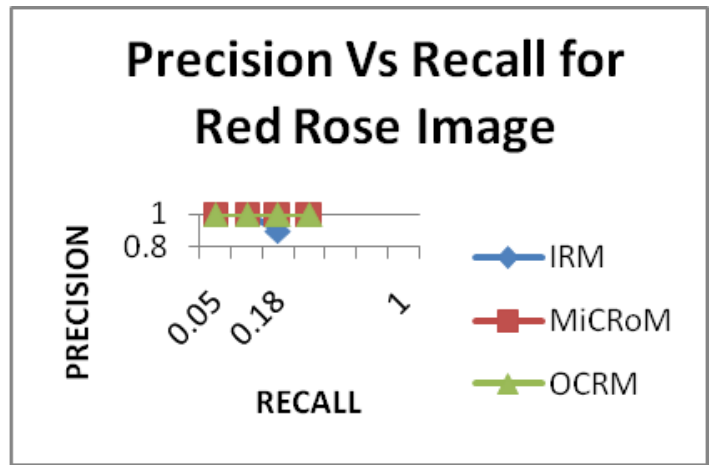

Figure 16. The Precision vs. Recall for the query image \#618(Red Rose)

\section{Conclusion}

The experimental results proved that the proposed OCRM method, and obtains optimum solutions with less number of iterations. Tables and graphs clearly indicate that for all images, 
the IRM gives comparatively less precision and recall value compared to that of MiCRoM and OCRM. Even though MiCRoM and OCRM are superior to that of IRM, OCRM is computationally economic than the other two. The OCRM similarity measure gives a metric distance, which is computationally inexpensive. This method successfully proved that after a rearrangement of regions in sorted order it always satisfies the monge condition. The NorthWest corner rule provides an optimum solution to the transportation problem only if the distance matrix fulfills the monge condition. This measure also satisfies with the human observation of similarity and is easily computable. As a result, the OCRM always obtains an optimal distance measure that is efficient and is computationally economic.

\section{References}

[1] M. Rehman, M. Iqbal, M. Sharif and M. Raza, "Content Based Image Retrieval: Survey" Word Applied Sciences Journal, vol. 19, no. 3, (2012), pp. 404-413.

[2] H. Yang and X. Zhou, "Research of Content Based Image Retrieval Technology", Proceedings of the Third International Symposium on Electronic Commerce and Security Workshops (ISECS 10) Guangzhou, P. R. China, vol. 29-31, (2010), pp. 314-316.

[3] R. Datta and D. Joshi, "Image Retrieval: Ideas, Influences, and trends øf the nelw age", In ACM Computing Surveys, vol. 40, no. 2, (2008).

[4] M. Flickner, et al., "Query by image and video content, the QBIC system", IEEE compute, vol. 28, no. 9, (1995), pp. 23-32.

[5] J. Li, J. Z. Wang and G. Wiederhold, "IRM: Integrated Region Matching for Image Retrieval", In Proc. of 8th ACM Int. Conf. on Multimedia, Los Angeles, California, (2000), pp. 147-156.

[6] R. O. Stehling, M. A. Nascimento and A X Falcao, "MiCRoM: A Metric Distance to Compare Segmented Images", In Proc. of the 2002 Visual (nfermation Systems Conference (VISUAL '02), (2002), pp. 2-23.

[7] H. A. Taha, "Operations Research", Ptentice Hall, (1982).

[8] S. T. Rachev, "Mass transportation problems theory" springer, vol. 1.

[9] R. E. Burkard, B. Klinz and R Rudolf, "Petspective of Monge properties in optimization", Discrete Applied Mathematics, vol. 70, (1990), pp. 95-161. )

[10] R. Rastogi and A. Natsêv, "WALRUS: A Similarity Retrieval Algorithm for Image Databases", SIGMOD, Philadelphia, PA, (1999).

[11] R. Shamir and B Dierieh, "Characerization and Algorithms for Greedily solvable Transportation Problems", Proc. 1st ACM/SIAMY Symposium onDiscrete Algorithms, (1993), pp. 358-366.

[12] M. A. Nascimento and V.Sridhar, "Effective and efficient region-based image retrieval", Journal of Visual Languages and Computing, vol, 14, (2003), pp. 151-179.

[13] A. K. Jain and R. C. Dubes, "Algorithms for Clustering Data”, Prentice Hall, New York, (1988).

[14] M. S. Kankanhalli, B M Mehtre and H. Y. Huang, "Color and Spatial Feature for Content-Based Image Retrieval”, Pattern Recognition Letters, vol. 20, (1999), pp. 109-118.

[15] B. G. Prasad, S. K. Gupta and K. K. Biswas, "Region-Based Image Retrieval Using Integrated Color Shape and Location Index", Computer Vision and Image Processing, vol. 94, (2004), pp. 193-233.

[16] I. Daubechies, "Ten Lectures on Wavelets", Capital City Press, (1992).

[17] J. A. Hartigan and M. A. Wong, "Algorithm AS136: a k-means clustering algorithm", Applied Statistics, vol. 28, (1979), pp. 100-108.

[18] J Li, J. Z. Wang and G. Wiederhold, "Classification of textured and non-textured images using region segmentation", Proceedings of the Seventh International Conference on Image Processing, Vancouver, BC, Canada, (2000) September.

[19] B. S. Manjunath, et al., "Color and texture descriptors", IEEE Transactions on Circuits and Systems for Video Technology, vol. 11, no. 6, (2001), pp. 703-715.

[20] C. Carson, M. Thomas, S. Belongie, J. M. Hellerstein and J. Malik, "Blobworld: a system for region-based image indexing and retrieval", Proceedings of the Third International Conference on Visual Information Systems (1999), , pp. 509-516.

[21] W.Y. Ma and B. S. Manjunath, "Netra: a toolbox for navigating large image databases", Multimedia Systems vol. 7, no. 3, (1999), pp. 184-198.

[22] J. Z. Wang, J. Li and G. Wiederhold, "Simplicity: semantics-sensitive integrated matching for picture libraries", IEEE Transactions on Pattern Analysis and Machine Intelligence, vol. 23, no. 9, (2001), pp. 947963. 
[23] R. K. Ahuja, T. L. Magnanti and J. B. Orlin, "Network Flows: Theory, Algorithms, and Applications", Prentice-Hall, Englewood Cliffs, NJ, (1993).

[24] Q. N. Huu, H. T. Thu and T. N. Quoc, "An Efficient Content Based Image Retrieval method for Retrieving Images", International Journal of Innovative Computing, information and Control, vol. 8, no. 4, (2012).

[25] Y. Rubner, C. Tomasi and L. J. Guibas, "The Earth Mover's Distance as a Metric for Image Retrieval", International Journal of Computer Vision, vol. 40, no. 2, (2000), pp. 99-121.

[26] T. Dharani and I. L. Aroquiaraj, “A survey on content based image retrieval”, Proceedings of the 2013 International Conference on Pattern Recognition, Informatics and Mobile Engineering (PRIME), Salem, India, (2013) February 21-22, pp: 485-490.

Authors

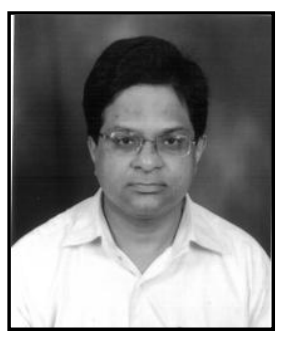

Vakulabharanam Vijaya Kumar received integrated M.S. Engg, degree from Tashkent Polytechnic Institute (USSR) in 1989. He received his $\mathrm{Ph} . \mathrm{D}$. degree in Computer Science from Jalwaharlal Nehru Technological University (JNTU) 1n 1998.He has served the JNT University for 13 years as As istant Professor and Associate Professor and taught courses for M.Tech students. He has been Dean for Dept of CSE and IT at Anurag Croup of Institutions. His research interests include Image Processing, Pattern Recognition, Network Security, and Steganography, Digital Watermarking, and Image retrieval. He is a life member for CSI, ISTE, IE, IRS, Aes and CS. He has published more than 100 research publications, in various National, Inter National conferences, proceedings and Jøurnals.

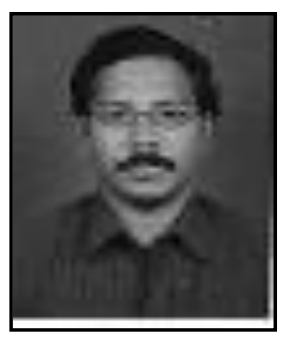

N.Gnaneswara Rao received B.E. (ECE) degree from Andhra University (S.R.K.R. Engineering College) in 1996 and received his M.Tech (CS) post graduation from the JNTU Hyderabad in 2002. He recieved PhD at JNTU Kakinada in the area of Content Based Image Retrieval He has working in Vignan University. He is a life member for CSI, TSTE, CRSI, IETE and a member of ACM, IET, and IEEE. He has published Ten research publications in various National, and Intecrational conferences

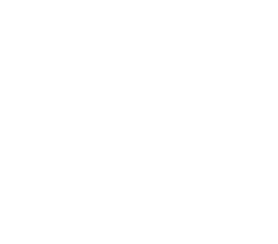


International Journal of Multimedia and Ubiquitous Engineering Vol.9, No.4 (2014)

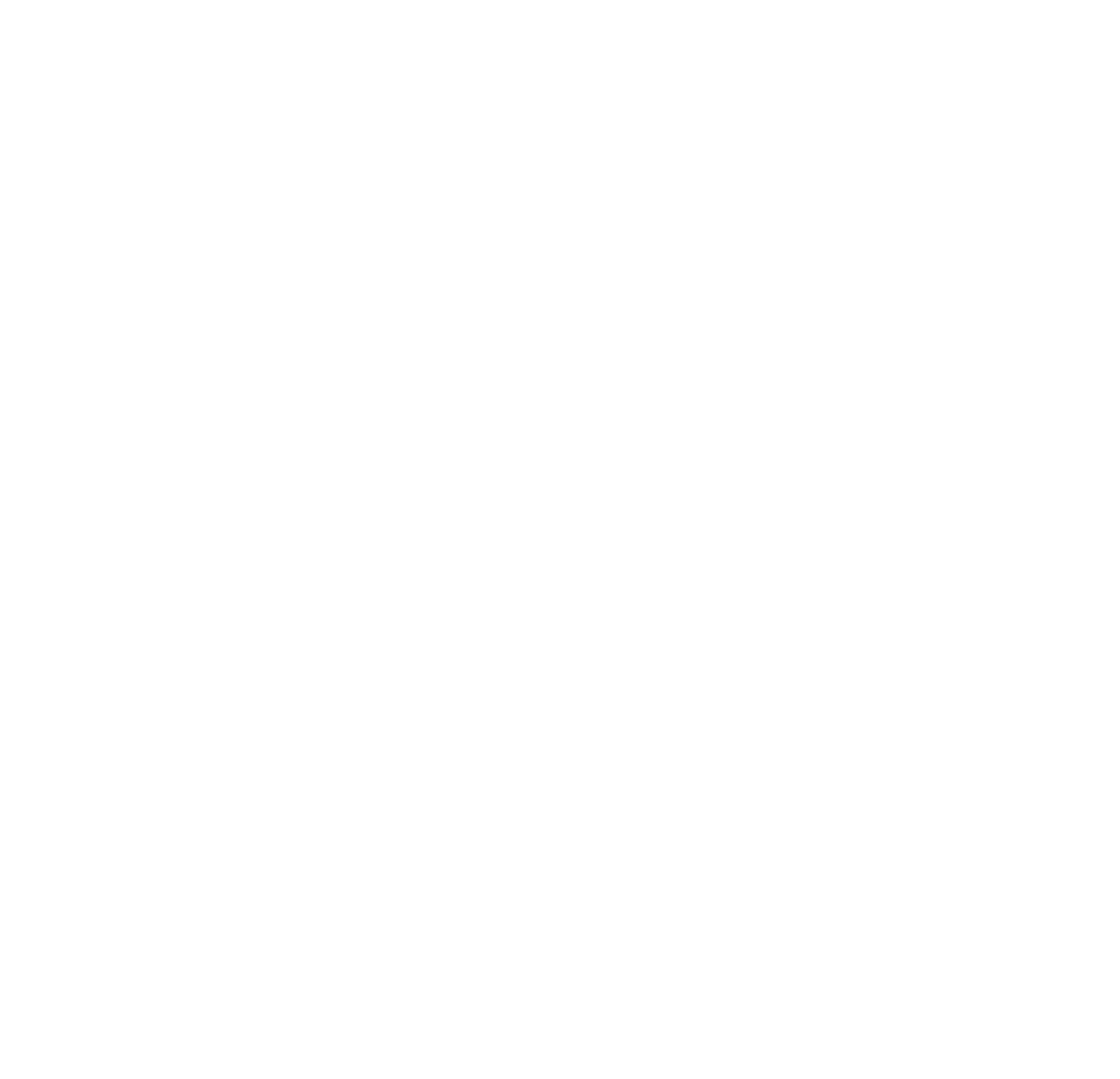

